\title{
201.
}

\section{SECOND NOTE ON THE THEORY OF LOGARITHMS.}

[From the Philosophical Magazine, vol. xII. (1856), pp. 354-360.]

THE theory of logarithms, as developed in my first note (Phil. Mag. April 1856), [197] may be exhibited in a clearer light by considering, instead of $\log a+\log b-\log a b=E \pi i$, the equation $\log \frac{b}{a}=\log b-\log a+E \pi i$, a form which more readily enables the accounting a priori for the discontinuity in the value of $E$. Writing then for $b, a$ the complex values $x^{\prime}+y^{\prime} i, x+y i$, we have

$$
\log \frac{x^{\prime}+y^{\prime} i}{x+y i}=\log \left(x^{\prime}+y^{\prime} i\right)-\log (x+y i)+E_{\pi i}
$$

where, according to the assumed definition of a logarithm,

$$
\log (x+y i)=\log \sqrt{x^{2}+y^{2}}+i\left(\tan ^{-1} \frac{y}{x}+\epsilon \pi\right)
$$

in which $\log \sqrt{x^{2}+y^{2}}$ is the real logarithm of $\sqrt{x^{2}+y^{2}}$, and $\tan ^{-1} \frac{y}{x}$ is an arc between the limits $-\frac{1}{2} \pi,+\frac{1}{2} \pi$. The coefficient $\epsilon$ is equal to zero when $x$ is positive; but when $x$ is negative, then $\epsilon=+1$ or -1 , according as $y$ is positive or negative, i.e. we have

$$
\begin{array}{ll}
x \equiv+, & \epsilon=0, \\
x \equiv-, \quad \epsilon= \pm 1 \equiv y
\end{array}
$$

and of course the other logarithms in the equation have an analogous signification. 
Hence, attending to the equation

$$
\tan ^{-1} \beta-\tan ^{-1} \alpha=\tan ^{-1} \frac{\beta-\alpha}{1+\alpha \beta}+\epsilon^{\prime \prime \prime} \pi,
$$

where, when $1+\alpha \beta$ is positive, $\epsilon^{\prime \prime \prime}$ is equal to zero; but when $1+\alpha \beta$ is negative, $\epsilon^{\prime \prime \prime}$ is equal to +1 or -1 , according as $\beta-\alpha$ is positive or negative: or what is the same thing ( $\alpha, \beta$ being of opposite signs when $1+\alpha \beta$ is negative),

we find

$$
\begin{array}{ll}
1+\alpha \beta \equiv+, & \epsilon^{\prime \prime \prime}=0, \\
1+\alpha \beta \equiv-, & \epsilon^{\prime \prime \prime}= \pm 1 \equiv \beta-\alpha \equiv \beta \equiv-\alpha,
\end{array}
$$

$$
E=\epsilon-\epsilon^{\prime}+\epsilon^{\prime \prime}-\epsilon^{\prime \prime \prime},
$$

where $\epsilon, \epsilon^{\prime}, \epsilon^{\prime \prime}, \epsilon^{\prime \prime \prime}$ are defined by the conditions

$$
\begin{array}{lll}
x & \equiv+, \quad \epsilon=0 \\
x & \equiv-, \quad \epsilon= \pm 1 \equiv y \\
x^{\prime} & \equiv+, \quad \epsilon^{\prime}=0 \\
x^{\prime} & \equiv-, \quad \epsilon^{\prime}= \pm 1 \equiv y^{\prime} \\
x x^{\prime}+y y^{\prime} \equiv+, & \epsilon^{\prime \prime}=0 \\
x x^{\prime}+y y^{\prime} \equiv-, & \epsilon^{\prime \prime}= \pm 1 \equiv x y^{\prime}-x^{\prime} y \\
1+\frac{y}{x} \frac{y^{\prime}}{x^{\prime}} \equiv+, & \epsilon^{\prime \prime \prime}=0 \\
1+\frac{y}{x} \frac{y^{\prime}}{x^{\prime}} \equiv-, & \epsilon^{\prime \prime \prime}= \pm 1 \equiv \frac{y^{\prime}}{x^{\prime}}-\frac{y}{x} \equiv \frac{y^{\prime}}{x^{\prime}} \equiv-\frac{y}{x} .
\end{array}
$$

Suppose, to fix the ideas, that $x, y$ are each of them positive, we have $\epsilon=0$; and considering the several cases:

1. $x^{\prime} \equiv+, \quad y^{\prime} \equiv+$.

Here $x x^{\prime}+y y^{\prime} \equiv+, 1+\frac{y}{x} \frac{y^{\prime}}{x^{\prime}} \equiv+;$ and consequently not only $\epsilon^{\prime}=0$, but also $\epsilon^{\prime \prime}=0$, $\epsilon^{\prime \prime \prime}=0$, and thence $E=0$.

2. $x^{\prime} \equiv+, \cdot y^{\prime} \equiv-$.

Here $\epsilon^{\prime}=0$. Moreover, $x x^{\prime}$ being positive, $x x^{\prime}+y y^{\prime}$ and $1+\frac{y}{x} \frac{y^{\prime}}{x^{\prime}}$ will have the same sign. If they are both positive, then $\epsilon^{\prime \prime}=0, \epsilon^{\prime \prime \prime}=0$; but if they are both negative, then

$$
\epsilon^{\prime \prime}= \pm 1 \equiv x y^{\prime}-x^{\prime} y \equiv \frac{y^{\prime}}{x^{\prime}}-\frac{y}{x}
$$

(since $x x^{\prime} \equiv+$ ) and $\epsilon^{\prime \prime \prime}= \pm 1 \equiv \frac{y^{\prime}}{x^{\prime}}-\frac{y}{x}$, i.e. $\epsilon^{\prime \prime}=\epsilon^{\prime \prime \prime}$. Hence in either case we have $E=0$. 
3. $x^{\prime} \equiv-, y^{\prime} \equiv+$.

Here $\epsilon^{\prime}= \pm 1 \equiv y^{\prime}$, i.e. $\epsilon^{\prime}=1$. Also $x x^{\prime}$ being negative; $x x^{\prime}+y y^{\prime}$ and $1+\frac{y}{x} \cdot \frac{y^{\prime}}{x^{\prime}}$ will have opposite signs. Suppose first $x x^{\prime}+y y^{\prime}$ is positive, then $\epsilon^{\prime \prime}=0$. And $1+\frac{y}{x} \frac{y^{\prime}}{x^{\prime}}$ being negative, we have $\epsilon^{\prime \prime \prime}= \pm 1 \equiv \frac{y^{\prime}}{x^{\prime}}-\frac{y}{x} \equiv \frac{y^{\prime}}{x^{\prime}}$, i.e. $\epsilon^{\prime \prime \prime}=-1$. But if $x x^{\prime}+y y^{\prime}$ is negative, then $\epsilon^{\prime \prime}= \pm 1 \equiv x y^{\prime}-x^{\prime} y \equiv-\left(\frac{y^{\prime}}{x^{\prime}}-\frac{y}{x}\right)$ (since $x x^{\prime}$ is negative) $\equiv-\frac{y^{\prime}}{x^{\prime}}$, i. e. $\epsilon^{\prime \prime}=+1 . \quad$ And $1+\frac{y}{x} \frac{y^{\prime}}{x^{\prime}}$ being positive, we have $\epsilon^{\prime \prime \prime}=0$. Hence in each case $E=0$.

4. $x^{\prime} \equiv-, y^{\prime} \equiv-$.

Here $\epsilon^{\prime}= \pm 1 \equiv y^{\prime}$, i.e. $\epsilon^{\prime}=-1$. Also $x x^{\prime}$ and $y y^{\prime}$ being each negative, $x x^{\prime}+y y^{\prime}$ will be negative, and therefore

$$
\epsilon^{\prime \prime}= \pm 1 \equiv x y^{\prime}-x^{\prime} y \equiv-\left(\frac{y^{\prime}}{x^{\prime}}-\frac{y}{x}\right)
$$

i.e. if $\frac{y^{\prime}}{x^{\prime}}>\frac{y}{x}$, then $\epsilon^{\prime \prime}=-1$; but if $\frac{y^{\prime}}{x^{\prime}}<\frac{y}{x}$, then $\epsilon^{\prime \prime}=+1$. And $1+\frac{y}{x} \frac{y^{\prime}}{x^{\prime}}$ being positive, $\epsilon^{\prime \prime \prime}=0$. Hence if $\frac{y^{\prime}}{x^{\prime}}>\frac{y}{x}$, then $E=1-1=0 ;$ but if $\frac{y^{\prime}}{x^{\prime}}<\frac{y}{x}$, then $E=1+1=2$.

Consider $(x, y)\left(x^{\prime}, y^{\prime}\right)$ as the rectangular coordinates of two points $A, A^{\prime}$. In the case which has been considered, the point $A$ has been taken in the positive quadrant; and the preceding discussion shows that we have always $E=0$, except in the case where the finite line $A A^{\prime}$ meets the negative portion of the axis of $x$, in which case we have $E=+2$. The same thing is true generally in whichever quadrant

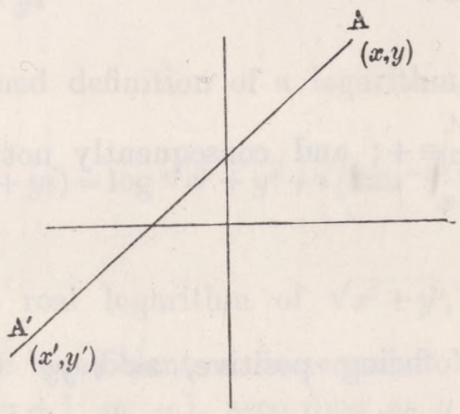

$A$ is situated, i.e. we have always $E=0$, except in the cases in which the finite line $A A^{\prime}$ meets the negative portion of the axis of $x$. But when this happens, then if the line $A A^{\prime}$, considered as drawn from $A$ to $A^{\prime}$, passes from above to below the axis of $x$, we have $E=+2$; but if the line $A A^{\prime}$, considered as drawn from $A$ to $A^{\prime}$, passes from below to above the axis of $x$, then $E=-2$. So that treating the points 
$A, A^{\prime}$ as the geometrical representations of the complex numbers $x+y i, x^{\prime}+y^{\prime} i$, we have in an exceedingly simple form the precise determination of the discontinuous number $E(=0$ or \pm 2$)$ in the formula

$$
\log \frac{x^{\prime}+y^{\prime} i}{x+y i}=\log \left(x^{\prime}+y^{\prime} i\right)-\log (x+y i)+E_{\pi i} .
$$

Consider in general the definite integral

$$
\int_{z}^{z^{\prime}} \phi u d u
$$

where $z^{\prime}, z$ are complex numbers of the form $x+y i, x^{\prime}+y^{\prime} i$; and take $A, A^{\prime}$ as the geometrical representations of these limits, and the variable point $P$ as the geometrical representation of the complex variable $u$. The value of the definite integral will depend to a certain extent on the series of values which we suppose $u$ successively to assume in passing from $z$ to $z^{\prime}$, or what is the same thing, on the path of the variable point $P$ from $A$ to $A^{\prime}$. For (excluding altogether the case in which the path passes through a point for which $\phi u$ becomes infinite) it is well known that the value of the definite integral is the same for any two paths which do not include between them a point for which $\phi u$ becomes infinite; but when this condition is not satisfied, then the value of the definite integral is not in general the same for the

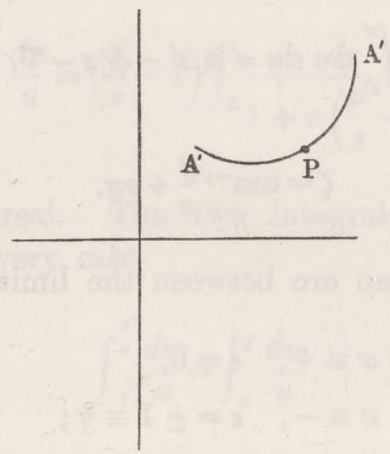

two paths $\left({ }^{1}\right)$. In order therefore to give a precise signification to the notations, we must fix the path of the point $P$, and it is natural to assume that the path is a right line (of course there are an infinity of paths which give the same value to the definite integral, or as we may call them, paths equivalent to the right line; but the consideration of these would be a needless complication of the definition, and it is better to attend to the single path-the right line). The definition is at once converted into an analytical one; we have only to assume $u=z+r\left(z^{\prime}-z\right)$, and to suppose that the new variable $r$ passes from $r=0$ (which gives $u=z$ ) through real

1 The theorem is, I believe, due to M. Cauchy. See the memoir of M. Puisenx, Recherches sur les Fonctions Algébriques, Liouville, vol. xv. [1850] pp. 365-480, where the subject is elaborately discussed.

C. III. 
values to $r=1$ (which gives $u=z^{\prime}$ ), i.e. we have as the equivalent analytical definition of the definite integral between the complex limits $z, z^{\prime}$ the equation

$$
\int_{z}^{z^{\prime}} \phi u d u=\left(z^{\prime}-z\right) \int_{0}^{1} \phi\left[z+r\left(z^{\prime}-z\right)\right] d r,
$$

where the new variable $r$ is real. The only restriction is, that $\phi u$ must not become infinite for any value of $u$ along the path in question, i.e. $\phi\left[z+r\left(z^{\prime}-z\right)\right]$ must not become infinite for any real value of $r$ between the limits $r=0, r=1$.

Suppose next, the path being defined as above, or in any other manner, that $\phi, u$ is a function of $u$ such that $\phi_{1}^{\prime} u=\phi u$. Then if $\phi, u$ is continuous along the entire path, we have

$$
\int_{z}^{z^{\prime}} \phi u d u=\phi, z^{\prime}-\phi, z
$$

but if $\phi, u$ is discontinuous at any points of the path, e.g. at the point $u=u_{,}$, and at no other point, then

$$
\int_{z}^{z^{\prime}} \phi u d u=\phi_{1} z^{\prime}-\phi_{1}\left(u,+\alpha^{\prime}\right)+\phi_{1}(u,-\alpha)-\phi_{1} z,
$$

where $u_{1}-\alpha_{1}, u_{1}+\alpha^{\prime}$ are values indefinitely near to $u_{1}$, the path being from $z$ through $u_{1}-\alpha$ to $u_{1}+\alpha^{\prime}$ and thence to $z^{\prime}$. Or if we represent the break $\phi_{1}\left(u,+\alpha^{\prime}\right)-\phi_{1}(u,+\alpha)$ by the symbol $\nabla$, then we have

$$
\int_{z}^{z^{\prime}} \phi u d u=\phi, z^{\prime}-\phi, z-\nabla
$$

Suppose now

$$
\zeta=\tan ^{-1} \frac{y}{x}+\epsilon \pi
$$

where, as before, $\tan ^{-1} \frac{y}{x}$ denotes an arc between the limits $-\frac{1}{2} \pi,+\frac{1}{2} \pi$, and

$$
\begin{array}{ll}
x \equiv+, & \epsilon=0, \\
x \equiv-, & \epsilon= \pm 1 \equiv y ;
\end{array}
$$

and to fix the ideas, consider $\zeta$ as the $z$-coordinate of a surface, the other two coordinates being $x$ and $y$. If $x$ be negative and $y$ be indefinitely small and positive, then $\epsilon=+1$, and we have $\zeta=\pi$; but if ( $x$ being still negative) $y$ be indefinitely small and negative, then $\epsilon=-1$, and therefore $\zeta=-\pi$, i.e. there is a break or abrupt increment $2 \pi$ of the coordinate $\zeta$ in passing across the negative part of the axis of $x$ from a negative to a positive value of $y$, or, as we have before called it, from below to above; this is the only discontinuity in the surface, the form of the surface being, in fact, what is intended to be represented in the annexed figure.

Suppose now $z^{\prime}=x^{\prime}+y^{\prime} i, z=x+y i$, and consider the definite integral

$$
\int_{z}^{z^{\prime}} \frac{d u}{u}
$$


the path being, as before, a right line. We have, by the equivalent analytical definition,

$$
\int_{z}^{z^{\prime}} \frac{d u}{u}=\left(z^{\prime}-z\right) \int_{0}^{1} \frac{d r}{z+r\left(z^{\prime}-z\right)}
$$

where the new variable $r$ is real. And in like manner considering the integral $\int_{1}^{\frac{z^{\prime}}{z}} \frac{d u}{u}$,

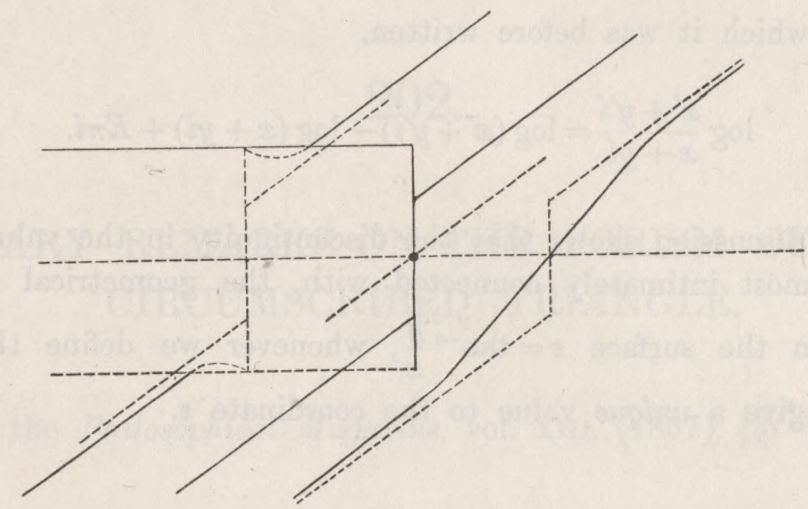

the path being in this case also a right line, we have

$$
\int_{1}^{z^{\prime}} \frac{d u}{u}=\left(\frac{z^{\prime}}{z}-1\right) \int_{0}^{1} \frac{d r}{1+r\left(\frac{z^{\prime}}{z}-1\right)}
$$

where the new variable $r$ is real. The two integrals in $r$ are identically the same, and consequently we have in every case

$$
\int_{1}^{\frac{z^{\prime}}{z}} \frac{d u}{u}=\int_{z}^{z^{\prime}} \frac{d u}{u}
$$

Now $\log ^{\prime} u=\frac{1}{u}$; and in passing from $u=1$ to $u=\frac{z^{\prime}}{z}$, there is no discontinuity in the value of $\log u$,

$$
=\log \sqrt{p^{2}+q^{2}}+i\left(\tan ^{-1} \frac{q}{p}+\epsilon \pi\right)
$$

if for the moment $u=p+q i$; hence the value of the integral on the left-hand side is simply $\log \frac{z^{\prime}}{z}$. The value of the integral on the right-hand side is in like manner $\log z^{\prime}-\log z$, in the case in which the finite right line from $u=z$ to $u=z^{\prime}$ does not meet the negative part of the axis of $x$; but when this happens, then there is a discontinuity in the value of the logarithm, and the integral on the right-hand side 
will be $\log z^{\prime}-\log z-2 \pi i$, or $\log z^{\prime}-\log z+2 \pi i$, according as the right line considered as drawn from $z$ to $z^{\prime}$ passes from below to above or from above to below the negative part of the axis of $x$. We have therefore in every case ( $E$ being defined as above) $\log z^{\prime}-\log z+E_{\pi i}$ for the value of the integral on the right-hand side, and the relation between the two integrals gives, as it ought to do, the equation

$$
\log \frac{z^{\prime}}{z}=\log z^{\prime}-\log z+E_{\pi i}
$$

or, in the form in which it was before written,

$$
\log \frac{x^{\prime}+y^{\prime} i}{x+y i}=\log \left(x^{\prime}+y^{\prime} i\right)-\log (x+y i)+E \pi i .
$$

The preceding discussion shows that the discontinuity in the value of $E(-0$ or \pm 2$)$ arises from, or is most intimately connected with, the geometrical discontinuity which necessarily exists in the surface $z=\tan ^{-1} \frac{y}{x}$, whenever we define the symbol $\tan ^{-1}$ in such manner as to give a unique value to the coordinate $z$.

2, Stone Buildings, Sept. 19, 1856. 